\title{
Effect of cultivar mixtures on the wave velocity of expanding yellow stripe rust foci in winter wheat
}

\author{
A. A. M. BUIEL ${ }^{1}$, M. A. VERHAAR ${ }^{1}$, F. VAN DEN BOSCH ${ }^{2}$, W. HOOG- \\ KAMER $^{1}$ \& J. C. ZADOKS ${ }^{1}$ \\ ${ }^{1}$ Department of Phytopathology, P.O. Box 8025, NL 6700 EE Wageningen, Netherlands \\ 2 Institute of Theoretical Biology, State University of Leiden, Groenhovenstraat 5, NL 2311 \\ BT Leiden, Netherlands
}

Received 8 September 1988; accepted 25 January 1989

\begin{abstract}
In a field experiment, the wave velocity of an expanding focus of yellow stripe rust (Puccinia striiformis) in four mixtures of two winter wheat cultivars was determined. Results obtained by two calculation methods yield a logarithmic relationship between the wave velocity and the proportion of susceptible plants.
\end{abstract}

Keywords: yellow stripe rust, Puccinia striiformis, focus expansion, wave velocity, cultivar mixture

\section{Introduction}

Focal expansion as described by Zadoks \& Schein (1979), reaches a constant velocity (wave velocity) after an initial phase of build-up (Kampmeijer \& Zadoks, 1977). Van den Bosch et al. (1988a, b) developed an analytical model which describes the radial expansion of plant disease foci and they examined the relation between various epidemiological parameters and the wave velocity of the expanding focus. According to their model (van den Bosch et al., 1988b), the velocity of the expanding focus increases logarithmically with the percentage of susceptible plants in a homogeneous mixture of susceptible and resistant plants.

The aim of the present study was to investigate this relationship experimentally for yellow stripe rust (Puccinia striiformis) of wheat, using mixtures of a susceptible and a resistant cultivar. Two methods for calculating the wave velocity were compared.

\section{Material and methods}

On 1987 January 30, the winter wheat cultivars 'Okapi' and 'Sarno', susceptible and resistant, respectively, were sown in pots in four mixtures, viz. Okapi pure $(1: 0)$, 
Okapi and Sarno in equal amounts (1:1), one-third Okapi and two-thirds Sarno (1:2), and one-fifth Okapi and four-fifths Sarno (1:4). Three square plots of each mixture were planted on 13 April in the South Flevopolder. The plots were separated from each other by at least $70 \mathrm{~m}$ of winter rape seed. A plot consisted of $11 \times$ 11 pots, planted at a centre-to-centre distance of $0.3 \mathrm{~m}$. Two pots of Okapi were inoculated in the greenhouse and planted in the centre of each plot to initiate the foci. The plants contained in one pot formed an experimental unit. The number of sporulating lesions on each unit was counted weekly. The course of the epidemic was registered until the pathogen disappeared, following the high day temperatures $\left(28^{\circ} \mathrm{C}\right)$ at the end of June.

In order to calculate the wave velocity of the expanding focus, two different methods were applied. The first method, hereafter called the gradient method, determined the wave velocity of the focus from the disease gradient which moved away from the centre with time. The velocity was calculated at three disease levels namely 1, 6 and 11 lesions per experimental unit. The second method, referred to as the area method, calculated the wave velocity from the enlargement of the diseased area (defined as area with experimental units carrying more lesions than a fixed level) with time. Here the wave velocity was calculated at two disease levels, of at least 6 and 11 lesions per experimental unit, respectively. The disappearance of lesions, a result of the shifting of the epidemical wave and the dying off of the lower leaves, would underestimate the velocity calculated by either method. Therefore, the cumulative number of lesions per experimental unit was evaluated.

\section{Results}

From the time the wave velocity had reached its constant value (5 June) until the breakdown of the epidemic due to high temperatures ( 25 June), the velocity was calculated per plot. Table 1 shows the wave velocity, $c$, and its standard deviation, s.d., per mixture averaged over disease levels and over plots. In both methods the wave velocity increases with an increase of the proportion of susceptible plants. The absolute values, however, differ considerably between methods. The s.d. of the gradient method was found to be much larger than the s.d. of the area method. The relative wave velocity, which is the measured wave velocity expressed as a fraction of the wave velocity in the $1: 0$ plots $\left(c / c_{\text {max }}\right)$, is plotted against the logarithm

Table 1. Mean wave velocity, $c$, and its standard deviation, s.d., of four wheat cultivar mixtures as assessed by the gradient method and the area method.

\begin{tabular}{|c|c|c|c|c|c|}
\hline \multirow{2}{*}{$\begin{array}{l}\text { Mixture } \\
\text { Okapi:Sarno }\end{array}$} & \multirow{2}{*}{$\begin{array}{l}\text { Susceptible } \\
\text { plants }(\%)\end{array}$} & \multicolumn{2}{|c|}{ Gradient method } & \multicolumn{2}{|l|}{ Area method } \\
\hline & & $\overline{c\left(\mathrm{~cm} \mathrm{day}^{-1}\right)}$ & $\overline{\text { s.d. }}$ & $\overline{c\left(\mathrm{~cm} \mathrm{day}^{-1}\right)}$ & s.d. \\
\hline $1: 0$ & 100.0 & 5.16 & 3.49 & 3.02 & 0.73 \\
\hline $1: 1$ & 50.0 & 4.26 & 1.44 & 1.99 & 0.76 \\
\hline $1: 2$ & 33.3 & 3.81 & 2.73 & 1.45 & 0.43 \\
\hline $1: 4$ & 20.0 & 2.46 & 1.13 & 0.74 & 0.37 \\
\hline
\end{tabular}




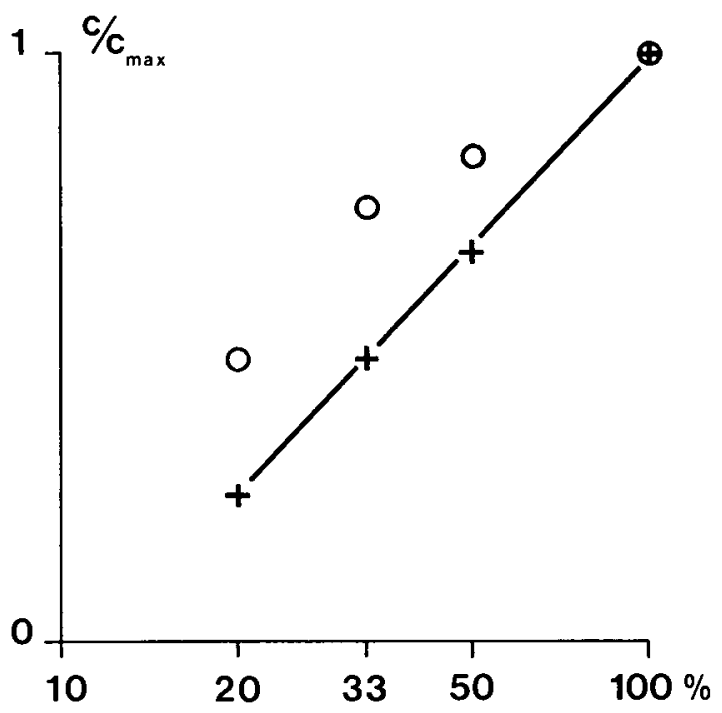

Fig. 1. The relative wave velocity, $c / c_{\max }$, as a function of the logarithm of the percentage of susceptible plants. $O$ Gradient method. + Area method.

of percentage of susceptible plants in Figure 1. The relative wave velocity appears to be proportional to the logarithm of the proportion of susceptible plants. The linear correlation coefficient is 0.98 for the gradient method and 1.00 for the area method.

\section{Discussion and conclusions}

The occurrence of exceptionally highly diseased plants in the edges of the plots, to which the gradient method is rather sensitive, led to an over-estimation of the wave velocity by the gradient method. The area method did not suffer from this disadvantage, as also evidenced by its lower variance and therefore the area method is recommended for calculating wave velocities.

The wave velocity of $3 \mathrm{~cm}$ day $^{-1}$ is rather low compared to $9 \mathrm{~cm} \mathrm{day}^{-1}$ found by van den Bosch et al. (1988c). This is probably due to unfavourable weather conditions for yellow stripe rust during this field experiment.

Both methods result in a logarithmic relationship between the wave velocity of the expanding focus and the proportion of susceptible plants. Accordingly, these data support the model of van den Bosch et al. (1988b).

\section{References}

Kampmeijer, P. \& J. C. Zadoks, 1977. EPIMUL, a simulator of foci and epidemics in mixtures, multilines, and mosaics of resistant and susceptible plants. Simulation Monographs, Pudoc, Wageningen. $50 \mathrm{pp}$. 
A. A. M. BUIEL ET AL.

Van den Bosch, F., J. C. Zadoks \& J. A. J. Metz, 1988a. Focus expansion in plant disease. I: The constant rate of focus expansion. Phytopathology 78: 54-58.

Van den Bosch, F., J. C. Zadoks \& J. A. J. Metz, 1988b. Focus expansion in plant disease. II: Realistic parameter-sparse models. Phytopathology 78: 59-64.

Van den Bosch, F., J. C. Zadoks \& J. A. J. Metz, 1988c. Focus expansion in plant disease. III: Two experimental examples. Phytopathology 78: 919-925.

Zadoks, J. C. \& R. D. Schein, 1979. Epidemiology and plant disease management. Oxford University Press, New York. 427 pp.

This synopsis is based on a doctoral thesis entitled 'Haarduitbreiding van gele roest in wintertarwe' (Focus expansion of yellow stripe rust in winter wheat) by H. Buiel and M. Verhaar, Department of Phytopathology, Wageningen Agricultural University, Wageningen, 1987, 61 pp., 12 figs., 9 tables, 19 refs., 11 appendices, Dutch. Available as paper copy (order R090, f 10 including postage) at: NARD, clo Pudoc, P.O. Box 4, 6700 AA Wageningen (telex 45015 blhwg nl). 